\title{
Perindopril treatment promote left ventricle remodeling in patients with heart failure screened positive for autoantibodies against angiotensin II type 1 receptor
}

Qian $\mathrm{Du}^{1 \dagger}$, Jinling $\mathrm{Wu}^{1 \dagger}$, Hua Wang ${ }^{1}$, Xin Wang $^{1}$, Lin Xu' ${ }^{1}$ Zhiyong Zhang ${ }^{1}$, Jiamei Liu', Juan Zhang ${ }^{1}$, Jin Chen ${ }^{1}$, Hakon Hakonarson ${ }^{2}$, Aihua $\mathrm{Hu}^{2}$ and Lin Zhang ${ }^{1 *}$

\begin{abstract}
Background: Autoantibodies specific to the angiotensin II type I receptor (anti-AT $T_{1}$-AR) have been implicated in the pathology of congestive heart failure (CHF). Anti-AT 1 -AR may be associated with left ventricular function in $\mathrm{CHF}$ patients treated with perindopril.

Methods: Synthetic angiotensin II type 1 receptor $\left(\mathrm{AT}_{1}-\mathrm{R}\right)$ peptides served as the target antigen. ELISA was used to screen the sera of 156 CHF patients, which were divided into positive and negative groups based on their anti-AT 1 -AR reactivity. Echocardiography and a 6-minute walk test were performed at baseline and after one year of perindopril therapy. The end-point events were compared over a 5-year follow-up.

Results: Final analysis covered 138 patients, including 82 positive and 56 negative. The frequency and geometric mean titre of anti-AT 1 -AR were significantly lower in the positive group after one year of treatment (all $P<0.01$, from $100 \%$ to 73.2\% and from 1:125.3 \pm 1.0 to 1:69.2 \pm 1.1 ). Of these, 22 patients showed no antibodies. Both groups showed improvement in left ventricular end-diastole, end-systolic dimensions, ejection fraction, and a 6 -minute walk test by perindopril in combination with standard treatment regime for one year (all $P<0.01$ ). However, the 82 patients positive for anti-AT 1 -AR showed more pronounced improvement than the 56 negative patients (all $P<0.05$ ). However, after 5 years of follow-up, the rate of all causes and cardiovascular mortality attributable to any cause and the re-hospitalisation rate showed no significant differences between the two groups (all $P>0.05$ ).

Conclusions: Perindopril treatment significantly decreased the frequency and geometric mean titre in patients positive for anti-AT 1 -AR, even to complete ablation. These patients showed greater improvement in left ventricular remodeling and heart function than negative that in patients after one year of perindopril treatment in combination with standard treatment, but no significant differences in endpoint events were observed in the following 5 years. Anti-AT 1 -AR might be a useful biomarker of over-activation of the renin-angiotensin-aldosterone system for clinical medication.
\end{abstract}

Keywords: Anti-AT 1 -AR, Renin-angiotensin-aldosterone system, Biomarker

\footnotetext{
* Correspondence: linzhangpeking@126.com

${ }^{\dagger}$ Equal contributors

'Heart Failure Center, Departments of Cardiology, Capital Medical University,

Chao-Yang Hospital, Beijing, China

Full list of author information is available at the end of the article
} 


\section{Background}

In recent years, there has been increased interest in autoimmune mechanisms involved in the pathogenesis of cardiovascular disease. Wallukat et al. demonstrated that the autoantibodies that activate angiotensin (Ang) II type 1 receptor (anti- $\mathrm{AT}_{1}-\mathrm{AR}$ ) are similar to those observed for the natural agonist Ang II [1,2]. A previous report by the present team showed that anti-AT ${ }_{1}-\mathrm{AR}$ exists in the sera of congestive heart failure (CHF) patients with ischemic cardiomyopathy and hypertension and that it may play an important role in the pathogenesis and myocardial remodeling of heart failure. Recently, Jin et al. found that anti-AT 1 - AR causes changes in cardiac tissue and function in rats [3]. In vitro, mouse cells experience an agonist effect similar to Ang II in cardiomyocyte hypertrophy [4].

Perindopril, a long-acting angiotensin-converting-enzyme inhibitor, can block the conversion of Ang I to Ang II and decrease the effects of Ang II that are mediated by $\mathrm{AT}_{1}$ receptor binding. Several studies have demonstrated that long-term administration of angiotensin-convertingenzyme inhibitor and $\beta$ - receptor blocker have additional therapeutic benefits [5,6]. However, the clinical value of anti- $\mathrm{AT}_{1}-\mathrm{AR}$ in heart failure patients and whether there are differential responses to perindopril between patients with and without anti-AT $\mathrm{A}_{1}-\mathrm{AR}$ remains unknown.

In the present study it is hypothesised that $\mathrm{CHF}$ patients with anti-AT $\mathrm{A}_{1}$-AR may experience more pronounced improvement in LV remodeling and heart function than patients without anti- $\mathrm{AT}_{1}-\mathrm{AR}$ in response to perindopril. Anti-AT ${ }_{1}$-AR may be useful as a marker for the pharmacological management in CHF patients.

\section{Methods}

\section{Study population}

This study was a prospective evaluation. The role of anti-AT 1 -AR was observed in CHF. From January 2005 to December 2010, $156 \mathrm{CHF}$ patients with dilated cardiomyopathy $(n=38)$, ischemic cardiomyopathy $(n=58)$, and hypertensive heart disease $(n=60)$ were recruited for the study. All patients were evaluated with respect to symptoms, physical examination, laboratory tests, chest radiography, cardiograms, and echocardiography. Each patient performed a minimum of two times of 6-minute walk tests, and the first scores of the pre- and posttreatment walk tests served as baseline values [7].

Criteria for enrolment were as follows: (1) age 1880 years old, (2) cardiac dysfunction caused by dilated cardiomyopathy, ischemic cardiomyopathy, or hypertensive heart disease, (3) stable New York Heart Association (NYHA) class II-IV heart function after treatment with $\beta$-receptor blocker, diuretics, and digoxin, (4) ability to complete the study visits, and (5) chronic cardiac insufficiency as defined by a LV ejection fraction (EF) $\leq 45 \%$. Data from hospital and physician records were analysed for adverse events such as re-hospitalisation, death from cardiovascular causes, and death from other causes. Exclusion criteria were as follows: (1) bilateral renal artery stenosis, unilateral renal artery stenosis, loss of function of kidney, cardiogenic shock, angiotensin-converting-enzyme inhibitor hypersensitivity, (2) hepatic dysfunction, (3) haemoglobin, creatinine, glutamic pyruvic transaminase, and potassium above or below the normal limit, creatinine

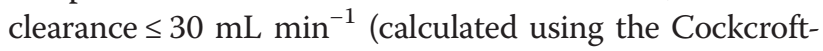
Gault formula), (4) stroke within the past 3 months, (5) systolic blood pressure over $160 \mathrm{mmHg}$ or diastolic blood pressure of more than $95 \mathrm{mmHg}$ despite antihypertensive therapy, and (6) terminal disease with a predicted survival time $<12$ months (e.g. terminal cancer). The study is compliant with the Declaration of Helsinki and was approved by the Ethics Committee and the Prescription and Therapeutic Committee of Beijing Chao-yang Hospital-Affiliate of Capital of Medical University. All patients in this study were given informed consent prior to enrolment in the study.

\section{Serum sampling and peptide synthesis}

Two millilitres of blood was drawn from the antecubital vein and separated by centrifugation (2000 rpm, Beckman CS-15R Centrifuge) for $10 \mathrm{~min}$. Serum samples were stored at $-20^{\circ} \mathrm{C}$ until needed for assay.

Peptides corresponding to the amino acid sequence (residues 165-191) of the second extracellular loop of the human $\mathrm{AT}_{1}$-AR (I-H-R-N-V-F-F-I-E-N-T-N-I-T-VC-A-F-H-Y-E-S-Q-N-S-T-L) were synthesised commercially (Genemed Synthesis Inc. CA, U.S.) [8]. The purity of the peptides was determined by high-performance liquid chromatography (HPLC) (Vydac C-18 column) and direct sequence analysis with an automated amino acid analyser (Beckman Instruments Inc, Palo Alto, CA, U.S.).

\section{ELISA-based screening for anti-AT 1 -AR}

Patients were defined as positive or negative subjects based on the presence or absence of anti- $\mathrm{AT}_{1}$-AR. An SA-ELISA protocol described previously was used to screen for the presence of the autoantibodies [8-11]. Briefly, $50 \mu \mathrm{L}$ of the peptide $\left(5 \mu \mathrm{g} \mathrm{mL}^{-1}\right)$ in $100 \mathrm{mM}$ $\mathrm{Na}_{2} \mathrm{CO}_{3}$ solution was coated on a microtitre plate overnight at $4^{\circ} \mathrm{C}$. The wells were saturated overnight with phosphate-buffered saline (PBS) supplemented with foetal bovine serum (FBS, 10\%, v/v). Serial dilutions of serum samples $(1: 20-1: 160)$ were then added to the antigen-coated microtitre plates and stored overnight at $4^{\circ} \mathrm{C}$. The blank group was given serum. An affinitypurified biotinylated rabbit antihuman $\lg \mathrm{G}(\mathrm{H}+\mathrm{L})$ antibody was allowed to react for $1 \mathrm{~h}$. The wells were washed three times (PBS, 10\% FBS and 0.1\% Tween$20 \mathrm{v} / \mathrm{v}$ ) and then incubated for $1 \mathrm{~h}$ with a streptavidin- 
peroxidase $\left(2 \mu \mathrm{L} \mathrm{mL} \mathrm{L}^{-1}\right)$ solution in PMT (PBS supplemented with $3 \%(\mathrm{wt} / \mathrm{vol})$ of skimmed milk, $0.1 \%(\mathrm{v} / \mathrm{v})$ of Tween-20 and $0.01 \%$ (wt/vol) of thimerosal (Sigma, St. Louis, MO, U.S.)). Incubation was followed by 3 rounds of washing with $\mathrm{PBS}$, after which a peroxidase substrate (2.5 mm $\mathrm{H}_{2} \mathrm{O}_{2}$-2 $\mathrm{mM} \mathrm{2,} \mathrm{2'-amino-di} \mathrm{(2-ethyl-benzo-}$ thiazoline sulphonic acid-6) ammonium salt (ABTS, Sigma, St. Louis, MO, U.S.) was added to the wells. The reaction was allowed to proceed for $30 \mathrm{~min}$ and then the sample's photometric qualities were evaluated (optical density, at $405 \mathrm{~nm}$ ) using a microplate reader (Labsystems Multiskan MK3, Helsinki, Finland).

Administration of angiogenesis-converting-enzyme inhibitor All patients received a standard pharmacological regimen for CHF (12.5-100 mg day ${ }^{-1}$ metoprolol, $10-20 \mathrm{mg}$ Qd spironolactone, and $0.125-0.25 \mathrm{mg}$ Qd digoxin) [12-14]. None of the patients included in this study had been treated with any angiotensin-converting-enzyme inhibitor before they enrolled in the study. Both groups received an initial daily dose of perindopril of $2 \mathrm{mg}^{\text {day }}{ }^{-1}$ (2 mg Qd), then up-titred over a 2-4 week period to a target dose of $4 \mathrm{mg} \mathrm{day}^{-1}$ (4 mg Qd). The criteria for increasing the dose included systolic blood pressure of $90 \mathrm{mmHg}$ or higher while the patient was standing, the absence of hypotension, and a serum creatinine concentration of either less than $2.0 \mathrm{mg}$ per decilitre $(177 \mu \mathrm{mol}$ per litre) or no more than $50 \%$ higher than the baseline concentration. The target heart rate and blood pressure were $55 \mathrm{bpm}$ and $90 / 60 \mathrm{mmHg}$, respectively. The $4 \mathrm{mg} \mathrm{day}^{-1}$ target dose was maintained until the termination of the study.

\section{Endpoint events and definitions}

The primary endpoint events were all-cause and cardiovascular mortality for a protocol-specific cardiovascular case. Re-hospitalisation for any cardiovascular event was the secondary endpoint. All endpoint events were adjudicated by members of an independent endpoint committee who were unaware of study group assignments and used pre-specified criteria.

\section{Follow-up}

Each patient was assigned to one designated study investigator from whom that patient received follow-up examinations at least once per month for 12 months after the initiation of the study, and then every 3 months for up to 5 years. Patients were encouraged to schedule interim appointments if needed. Data collected during patient examinations included heart rate, blood pressure, weight, presence of rales during the pulmonary exam, cardiac function, presence of peripheral edoema, and drug doses. Subjects were questioned and examined for the presence of any adverse drug reaction.

\section{Data analysis}

All data except antibody titres are presented as mean values $\pm \mathrm{SEM}$. An anti- $\mathrm{AT}_{1}$-AR positive score was defined as a ratio $\mathrm{P} / \mathrm{N}$ (sample $\mathrm{OD}$ - blank $\mathrm{OD} /$ negative control OD - blank OD) of $\geq 2.1$. Negative scores were defined as a ratio $\mathrm{P} / \mathrm{N}<2.1$. Antibody titres are reported as geometric means and differences between data sets were evaluated by unpaired Student's t-tests.

All other data were evaluated by Fisher exact test measures. In all cases, a $P$-value of $<0.05$ was considered statistically significant, and survival analysis was performed over 5 years. Kaplan-Meier curves were analysed over time to determine probability of survival using the log rank test according to the presence or absence of these autoantibodies. Analyses were performed using the GraphPad 5.0 software package (San Diego, CA, U.S.).

\section{Results}

\section{Clinical data and characteristics}

The patients were assigned to the positive and negative groups based on anti-AT ${ }_{1}$-AR reactivity, 88 to the positive group and 68 to the negative group. Six patients in the positive group and twelve patients in the negative group were not enrolled in the study. Of these, three patients positive for anti-AT $-\mathrm{AR}$ and four patients negative for anti-AT 1 -AR were not enrolled because their LVEF values improved to $>45 \%$ after standard pharmacological intervention. One patient with (-) anti$\mathrm{AT}_{1}$ - $\mathrm{AR}$ experienced sudden death, two patients with (+) anti-AT ${ }_{1}-\mathrm{AR}$ and seven patients with (-) anti- $\mathrm{AT}_{1^{-}}$ $\mathrm{AR}$ withdrew, and one patient with $(+)$ anti- $\mathrm{AT}_{1}-\mathrm{AR}$ ceased contact with the study personnel. Finally, there were 138 patients in this study, 82 patients with $(+)$ anti$\mathrm{AT}_{1}-\mathrm{AR}$ and 56 patients with $(-)$ anti-AT $\mathrm{T}_{1}-\mathrm{AR}$. The mean disease history (years) of $(+)$ anti-AT $\mathrm{AT}_{1}$-AR patients were shorter than those of $(-)$ anti-AT ${ }_{1}-\mathrm{AR}$ patients $(P<0.01,6.1 \pm 0.3$ vs. $8.7 \pm 0.4$ years $)$. Other baseline clinical characteristics remained consistent across all patients in the study (Table 1).

\section{Frequency of anti-AT $1-A R$ in the positive group}

The frequency of anti-AT $\mathrm{A}_{1}$-AR decreased significantly in the positive group $(P<0.01$, from $100 \%$ to $73.2 \%)$. Twenty two patients demonstrated complete ablation of anti-AT ${ }_{1}$-AR titres one year after the initiation of perindopril treatment. The geometric mean titre of the remaining 60 patients in the $(+)$ anti- $\mathrm{AT}_{1}$ - $\mathrm{AR}$ group was significantly lower than baseline values. The geometric mean titres were reduced as follows: Twenty one patients went from 1:160 to 1:80; ten patients went from 1:160 to 1:40; four patients went from 1:160 to $1: 20$; and two patients went from 1:80 to 1:40; one patient went from 1:80 to $1: 20$, and two patients went from 1:40 to 1:20. However, the remaining 20 patients experienced no 
Table 1 Clinical characteristics of patients (means \pm SEM)

\begin{tabular}{|c|c|c|c|}
\hline Index & $\begin{array}{c}(+) \text { anti-AT } 1-A R \\
(n=82)\end{array}$ & $\begin{array}{c}(-) \text { anti-AT } 1-A R \\
(n=56)\end{array}$ & $P$-value \\
\hline \multicolumn{4}{|l|}{ Demographic } \\
\hline Age (years) & $66.6 \pm 1.2$ & $65.1 \pm 1.6$ & ns \\
\hline Sex (male) & $42(51.2 \%)$ & $30(53.6 \%)$ & ns \\
\hline BMI $\left(\mathrm{kg} / \mathrm{m}^{2}\right)$ & $24.3 \pm 0.9$ & $24.3 \pm 0.6$ & ns \\
\hline Disease history (years) & $6.1 \pm 0.3$ & $8.7 \pm 0.4$ & $<0.01$ \\
\hline \multicolumn{4}{|l|}{ Aetiology } \\
\hline CAD cases $(\%)$ & $24(29.3 \%)$ & $26(46.4 \%)$ & ns \\
\hline DCM cases (\%) & $26(31.7 \%)$ & $8(14.3 \%)$ & ns \\
\hline HHD cases (\%) & $32(39.0 \%)$ & $22(39.3 \%)$ & ns \\
\hline \multicolumn{4}{|l|}{$\begin{array}{l}\text { Physiological } \\
\text { characteristics }\end{array}$} \\
\hline Heart rate (bpm) & $94.2 \pm 1.2$ & $93.8 \pm 1.2$ & ns \\
\hline SBP $(\mathrm{mmHg})$ & $129.9 \pm 1.8$ & $125.0 \pm 1.8$ & ns \\
\hline $\mathrm{DBP}(\mathrm{mmHg})$ & $78.2 \pm 0.9$ & $75.5 \pm 1.1$ & ns \\
\hline LVEDD (mm) & $69.5 \pm 0.6$ & $69.9 \pm 0.9$ & ns \\
\hline LVESD (mm) & $56.2 \pm 0.6$ & $55.7 \pm 0.8$ & ns \\
\hline LVEF (\%) & $34.1 \pm 0.6$ & $33.8 \pm 0.9$ & ns \\
\hline 6 min walk test (m) & $303.8 \pm 3.7$ & $301.8 \pm 4.3$ & ns \\
\hline
\end{tabular}

CAD: coronary artery disease; DCM: dilated cardiomyopathy; HHD: hypertensive heart disease; SBP: systolic blood pressure; DBP: diastolic blood pressure; LVEDD: left ventricular end-diastolic dimension; LVESD: left ventricular end-systolic dimension; LVEF: left ventricular ejection fraction.

change (Table 2). The current results provide clear clinical evidence that the anti- $\mathrm{AT}_{1}-\mathrm{AR}$ produced by $\mathrm{CHF}$ patients is suppressed by treatment with perindopril.

\section{Echocardiography and the 6-minute walk test}

After one year of perindopril treatment in combination with standard treatment for CHF ( $\beta$-receptor blockers, digoxin, and diuretics), LVEDD and LVESD values decreased from $69.5 \pm 0.6$ to $58.0 \pm 0.8 \mathrm{~mm}$ and $56.2 \pm 0.6$ to $42.0 \pm 0.7 \mathrm{~mm}$, respectively, in patients with $(+)$ anti$\mathrm{AT}_{1}-\mathrm{AR}$ and from $69.5 \pm 0.6$ to $58.0 \pm 0.8 \mathrm{~mm}$ and $56.2 \pm$ 0.6 to $42.0 \pm 0.7 \mathrm{~mm}$, respectively, in patients with $(-)$ anti-AT ${ }_{1}$-AR. Similarly, LVEF increased from $34.1 \pm 0.6$ to

Table 2 Perindopril and frequency and titre of anti-AT 1 -AR

\begin{tabular}{|c|c|c|c|}
\hline & $\begin{array}{l}\text { Pre-treatment } \\
\qquad \mathrm{n}=\mathbf{8 2}\end{array}$ & $\begin{array}{l}\text { Post-treatment } \\
\qquad n=58\end{array}$ & $P$-value \\
\hline $\begin{array}{l}\mathrm{AT}_{1} \text { autoantibody } \\
\text { frequency (\%) }\end{array}$ & $100 \%$ & $70.7 \%$ & $<0.01$ \\
\hline Mean titre & $125.3 \pm 1.0$ & $69.2 \pm 1.1$ & $<0.01$ \\
\hline Titre $1: 160$ & 55 & 13 & $<0.01$ \\
\hline Titre $1: 80$ & 25 & 27 & ns \\
\hline Titre $1: 40$ & 2 & 12 & ns \\
\hline Titre $1: 20$ & 0 & 6 & ns \\
\hline
\end{tabular}

Mean titre values are presented as geometric means \pm SEM.
$49.6 \pm 1.2 \%$ in patients with $(+)$ anti- $\mathrm{AT}_{1}-\mathrm{AR}$ and from $33.8 \pm 0.9$ to $44.3 \pm 1.1 \%$ in patients with $(-)$ anti-AT ${ }_{1}$-AR (all $P<0.01$ ).

It is worth noting that patients with $(+)$ anti- $\mathrm{AT}_{1}-\mathrm{AR}$ showed greater improvements with respect to in LV remodeling and heart function than patients with $(-)$ anti-AT ${ }_{1}$-AR (all $P<0.05$ ), as shown in Table 3.

The 6-minute walk distance scores of study participants at the beginning and experimental endpoint of the investigation were illustrated. Perindopril in combination with standard treatment was associated with significant increases in the distance covered in a timed 6-minute walk in both groups. The patients with $(+)$ anti-AT 1 -AR distance walked increased from $303.8 \pm 3.7$ to $488.6 \pm$ $11.2 \mathrm{~m}(P<0.01)$ and the patients with $(-)$ anti- $\mathrm{AT}_{1}-\mathrm{AR}$ was from $301.7 \pm 4.3$ to $452.9 \pm 9.2 \mathrm{~m}(P<0.01)$, but the former group showed more pronounced improvement $(P<0.05,488.6 \pm 11.2$ vs. $452.9 \pm 9.2 \mathrm{~m})$. Clinical lab data including haemoglobin, creatinine, glutamic pyruvic transaminase, and potassium levels remained stable throughout the first year.

\section{Tolerability and maximal titration of perindopril}

During the one-year treatment period, the mean perindopril doses for individuals the $(+)$ anti-AT $1-\mathrm{AR}$ and $(-)$ anti- $\mathrm{AT}_{1}$-AR were $3.3 \pm 0.1 \mathrm{mg}$ per/day and $2.9 \pm$ $0.1 \mathrm{mg}$ per/day $(P<0.05)$, respectively. Perindopril $(4 \mathrm{mg})$ was administered to at least $64.6 \%(53 / 82)$ of $(+)$ anti- $\mathrm{AT}_{1}-\mathrm{AR}$ patients, significantly more than the number of $(-)$ anti-AT ${ }_{1}$-AR patients $(44.6 \%, 25 / 56, P<0.05)$. These results suggested that the patients with $(+)$ anti$\mathrm{AT}_{1}$-AR demonstrated a greater tolerance to perindopril than those who did not express the autoantibodies (Figure 1).

\section{Primary endpoint events}

Mortality from any cause, cardiovascular mortality, and the re-hospitalisation rate in the $(+)$ anti- $\mathrm{AT}_{1}$ - $\mathrm{AR}$ group were $17.1 \%(14 / 82), 30.5 \%(25 / 82)$, and $14.4 \%(20 / 82)$ respectively. These values were not significant different from the $19.6 \%$ (11/56), 26.8\% (15/56), and 21.4\% (12/56) recorded

\section{Table 3 Perindopril and echocardiographic data}

\begin{tabular}{lccccc}
\hline & \multicolumn{2}{c}{$(+)$ anti- $\mathbf{A T}_{\mathbf{1}}$-AR $(\mathbf{n}=\mathbf{8 2})$} & & \multicolumn{2}{c}{$(-)$ anti- $\mathbf{A T}_{\mathbf{1}}$-AR $(\mathbf{n}=\mathbf{5 6})$} \\
\cline { 2 - 3 } \cline { 5 - 6 } & Baseline & One-year & & Baseline & One-year \\
\hline $\operatorname{LVEDD~}(\mathrm{mm})$ & $69.5 \pm 0.6$ & $58.0 \pm 0.8^{*} \dagger$ & & $69.9 \pm 0.9$ & $61.0 \pm 0.6^{*}$ \\
$\operatorname{LVEDD}(\mathrm{mm})$ & $56.2 \pm 0.6$ & $42.0 \pm 0.7^{*} \dagger$ & & $55.7 \pm 0.8$ & $47.1 \pm 1.0^{*}$ \\
$\operatorname{LVEF}(\%)$ & $34.1 \pm 0.6$ & $49.6 \pm 1.2^{*} \dagger$ & & $33.8 \pm 0.9$ & $44.3 \pm 1.1^{*}$ \\
\hline
\end{tabular}

LVEDD, left ventricular end-diastolic dimension; LVESD, left ventricular end-systolic dimension; LVEF, left ventricular ejection fraction. Both groups of patients showed significant improvement in response to perindopril therapy. Of note, the presence of $(+)$ anti-AT $1-A R$ group experienced more pronounced benefits than the (-) anti-AT 1 -AR group. Data were collected and analysed as described in the text. ${ }^{*} P<0.01$ vs. baseline and $+P<0.05$ vs. (-) anti-AT $1-\mathrm{AR})$. 


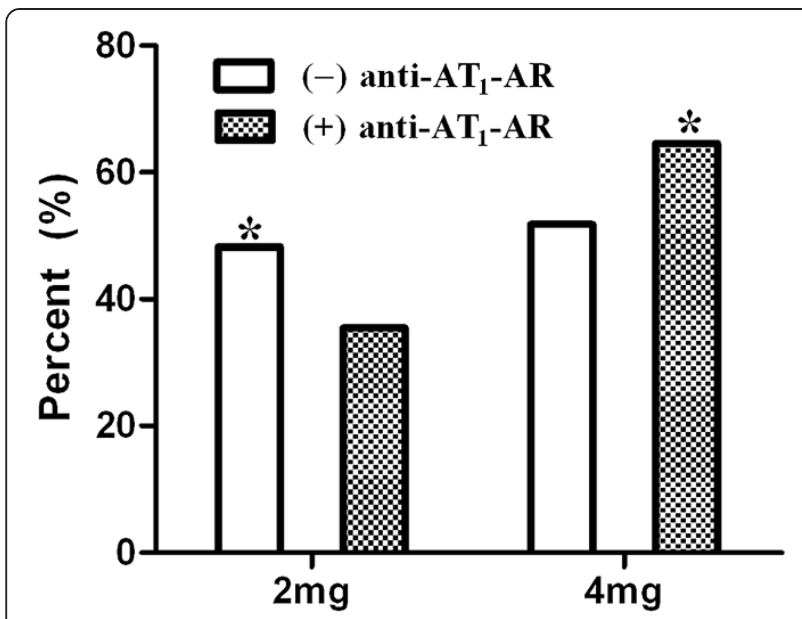

Figure 1 Perindopril dosage. During the one-year treatment period, the mean perindopril dose in the (+) anti-AT 1 -AR and (-) anti-AT 1 -AR group was $3.3 \pm 0.1 \mathrm{mg}$ Qd /day and $2.9 \pm 0.1 \mathrm{mg}$ $\mathrm{Qd} /$ day $(P<0.05)$, respectively. Perindopril $(4 \mathrm{mg})$ was administered to patients with $(+)$ anti-AT 1 -AR at significantly higher doses than patients with ( - ) anti-AT 1 -AR $(P<0.05,64.6 \%$ vs. $44.6 \%)$. These results suggest that the patients with $(+)$ anti-AT 1 -AR are more tolerant to perindopril than those that do not express these autoantibodies.

for the $(-)$ anti-AT ${ }_{1}$-AR group $(P=0.58, P=0.65$, and $P=0.66$ respectively), as shown in Figure 2 .

\section{Adverse effects}

One year of treatment with perindopril showed no significant effect on liver or kidney function. Three subjects became lethargic during the course of incremental administration of perindopril. However, these symptoms subsided as treatment continued. At least 3.6\% (5/138) of the patients developed a cough due to perindopril and were then transitioned to angiotensin receptor antagonist therapy with losartan.

\section{Discussion}

\section{Main findings}

In this study, three novel observations were made. First, the frequency and geometric mean titre of anti- $\mathrm{AT}_{1}-\mathrm{AR}$ deceased significantly in positive group. Patients with $(+)$ anti- $\mathrm{AT}_{1}-\mathrm{AR}$ showed greater improvements in $\mathrm{LV}$ remodeling and heart function than patients without anti-AT $T_{1}-\mathrm{AR}$. Second, patients with $(+)$ anti-AT $-A R$ were able to tolerate higher doses of perindopril than patients with (-) anti-AT ${ }_{1}$-AR during the first year of follow-up. Third, no statistically significant difference was detected between the two groups in terms of mortality from all cause, cardiovascular mortality, or rehospitalisation after five years of follow-up. These results suggested that perindopril may partially and selectively inhibit or block over-activation of $\mathrm{AT}_{1}$-receptor by anti$\mathrm{AT}_{1}$-AR. Furthermore, the long-term efficacy of perindopril in patients with $(+)$ anti-AT $1-\mathrm{AR}$ was found to be equivalent to those patients with (-) anti- $\mathrm{AT}_{1}-\mathrm{AR}$, and the reasons for this may involve patients with $(+)$ anti$\mathrm{AT}_{1}$ - $\mathrm{AR}$ becoming negative.

\section{Role of anti-AT ${ }_{1}-\mathrm{AR}$ in heart failure}

In recent years, evidence showing that an autoimmune mechanism might contribute to the pathogenesis of heart failure has accumulated. It has been reported that anti- $\mathrm{AT}_{1}-\mathrm{AR}$, anti- $\beta_{1}-\mathrm{AR}$, and anti- $\mathrm{M}_{2}-\mathrm{R}$ are present in sera of patients with a variety of cardiovascular diseases $[9,10,15]$. Recently Jin et al. found that, in rats, anti-AT ${ }^{-}$ AR could cause changes in cardiac tissue and function and that it exhibited an agonist effect in vitro similar to Ang II in cardiomyocyte hypertrophy [3]. Anti- $\mathrm{AT}_{1}-\mathrm{AR}$ is involved in the pathogenesis of cardiovascular diseases. In this way, the elimination of these antibodies might have therapeutic benefit for CHF patients.

\section{Anti-AT ${ }_{1}$-AR and activation of RAAS}

CHF is attributed to the long-term effects of cardiac ventricular reconstitution and excessive activation by neuroendocrine hormones, especially in the renin-angiotensin-aldosterone system (RAAS) [16]. Some studies have shown that cardiac $\mathrm{AT}_{1}-\mathrm{AR}$ is the primary receptor behind the regulation of heart function and that it mediates most of the known chronotropic and inotropic effects
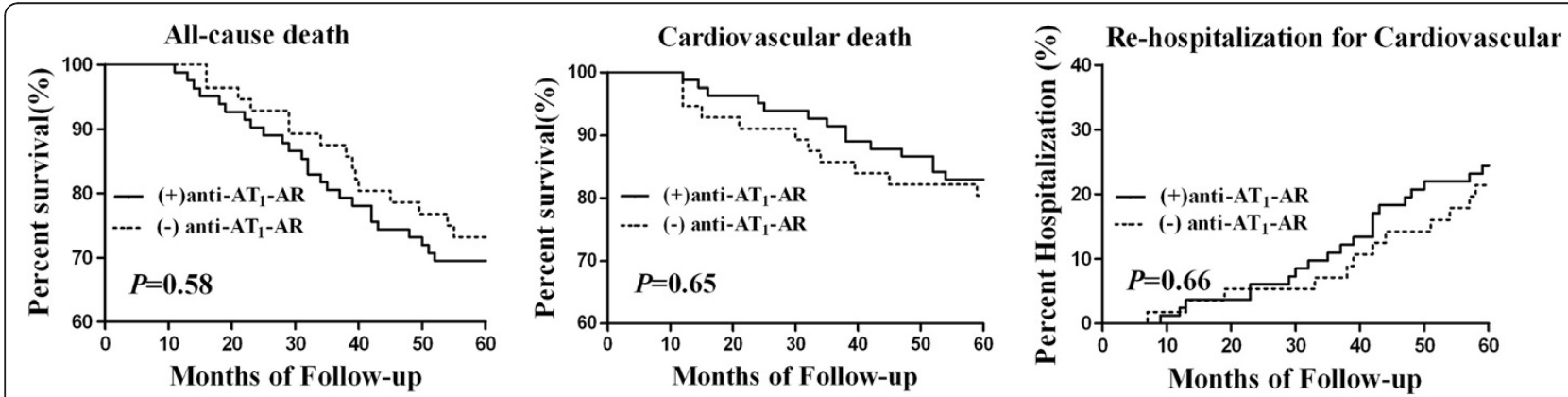

Figure $\mathbf{2}$ Endpoint events in both groups over $\mathbf{5}$ years. There was no significant difference in total mortality, cardiovascular mortality, or re-hospitalisation for a protocol-specific cardiovascular cases between patients with (+) anti-AT 1 -AR and (-) anti-AT 1 -AR (all $P>0.05)$. 
of Ang II in the heart, presynaptic facilitation of noradrenaline release from cardiac sympathetic nerve terminals, coronary vessel vasoconstriction, stimulation of aldosterone release, myocyte hypertrophy, non-myocyte proliferation, and interstitial fibrosis $[17,18]$. Anti- $\mathrm{AT}_{1}$-AR has also been showed to perform functions similar to those of Ang II $[19,20]$. The production of anti-AT ${ }_{1}-\mathrm{AR}$ may modulate the pathophysiological process of CHF by activating the RAAS and promoting ventricular remodeling in an angiotensin-II-independent manner. The binding of anti$\mathrm{AT}_{1}-\mathrm{AR}$ to $\mathrm{AT}_{1}-\mathrm{AR}$ can activate cell signalling pathways involved in cell damage, proliferation, and other pathophysiological processes [21]. One recent study demonstrated anti- $\mathrm{AT}_{1}-\mathrm{AR}$ can induce apoptosis of neonatal rat cardiomyocytes in a dose- and time-dependent manner. Anti-AT ${ }_{1}$-AR has been shown to increase TNF secretion and caspase- 3 activity. $\mathrm{AT}_{1}$ receptor blockage completely abrogated anti- $\mathrm{AT}_{1}$-AR-induced TNF- $\alpha$ secretion, caspase-3 activation, and cardiomyocyte apoptosis [22]. The existence of an autoimmune-activating receptor, such as anti-AT $\mathrm{A}_{1}-\mathrm{AR}$, could contribute to Ang II sensitivity [23]. These parameters may also be useful in the diagnosis and assessment of the pathogenesis of heart failure and in the stratification and identification of patients who would be at higher risk of heart failure or more responsive to therapeutic agents $[24,25]$.

\section{Perindopril and the effects of anti-AT 1 -AR}

Perindopril belongs to the angiotensin-converting enzyme (ACE) inhibitor class of medications. It is a potent, competitive inhibitor of $\mathrm{ACE}$, the enzyme responsible for the conversion of angiotensin $\mathrm{I}\left(\mathrm{AT}_{\mathrm{I}}\right)$ to angiotensin II $\left(\mathrm{AT}_{\mathrm{II}}\right)$, which regulates blood pressure and heart function. $\mathrm{AT}_{\mathrm{II}}$ is a key component of the renin-angiotensinaldosterone system (RAAS). It is here speculated that perindopril may be directly or indirectly related to the inhibition of over-activity of RAAS. Perindopril may decrease Ang II and increase Ang-(1-7) levels and so may attenuate immune responses to reduce or abolish the anti-AT ${ }_{1}$-AR effects. Angiotensin Ang-(1-7) is a biologically active peptide of the renin-angiotensin system whose actions are often opposite to those attributed to Ang II. This peptide may play a part in the beneficial effects of angiotensin-converting enzyme inhibitors in cardiovascular disease [26]. Tallant et al. reported that Ang-(1-7) inhibits the growth of cardiomyocytes and is associated with reduced activity of MAP kinase. These responses are opposite those of Ang II-AT ${ }_{1}$ activation. Cardiofibroblasts and Ang-(1-7) may underlie the antiinflammatory actions of the peptide that reverse cardiac fibrosis in the DOCA-salt rat model [27]. Ang-(1-7) induce vasodilatation, exert antiproliferative effects in vascular smooth muscle cells, and increase the sensitivity of the baroreflex.
Extensive studies have shown that $\mathrm{AT}_{2}$-receptor is re-expressed by cardiac fibroblasts during heart failure. Direct $\mathrm{AT}_{2}$ receptor stimulation was found to induce anti-inflammatory and anti-oxidant effects and was involved in anti-remodeling mechanisms that improved cardiac function [28,29]. It is here speculated that anti$\mathrm{AT}_{1}-\mathrm{AR}$ and Ang II may stimulate re-expression of the $\mathrm{AT}_{2}$ receptor in some situations to some extent and that this might have therapeutic effects. Moreover, in primary aldosteronism, after challenge with ACE inhibitors, the plasma aldosterone concentration fell more in anti- $\mathrm{AT}_{1}$ AR positive individuals than in negative individuals, suggesting that this autoantibody plats an agonistic role [30]. ACE inhibitors may reduce plasma aldosterone through anti-AT $\mathrm{T}_{1}-\mathrm{AR}$ agonism. Therapies for heart failure and activation of the immune system may improve as drug standardisation becomes more sophisticated, and antibody titre should decrease.

\section{Predictive value of anti-AT 1 -AR}

Continual activation of autoantibodies may lead to a poor prognosis. Studies have shown that stimulating autoantibodies against the cardiac $\beta 1$-adrenergic receptor can predict increased mortality among individuals with idiopathic cardiomyopathy [31]. It is here suggested that perindopril may weaken or even eliminate the adverse effects of anti-AT 1 -AR during the initial stage of CHF. This might be mediated by activating an immune mechanism that can weaken or offset CHF and ventricular remodeling and so improve cardiac function. In this way, long-term administration of perindopril in conjunction with digoxin, diuretics, and beta-blockers may prevent over-stimulation of $\mathrm{AT}_{1}-\mathrm{AR}$ by anti- $\mathrm{AT}_{1}-\mathrm{AR}$ and reduce the progression of cardiac damage. In addition, perindopril, which is an angiotensin-converting-enzyme inhibitor, can decrease plasma aldosterone levels and improve the prognosis of patients with severe CHF by decreasing plasma rennin-angiotensin levels [32]. In this way, anti- $\mathrm{AT}_{1}-\mathrm{AR}$ may be used to predict treatment response to select angiotensin-converting-enzyme inhibitors in patients with CHF. The specific pathological mechanism of anti- $\mathrm{AT}_{1}-\mathrm{AR}$ requires further exploration.

\section{Conclusions}

The results of this study suggest that the frequency and geometric mean titre in patients with $(+)$ anti- $\mathrm{AT}_{1}-\mathrm{AR}$ decreases significantly, even complete ablation. Patients positive for anti-AT $\mathrm{T}_{1}-\mathrm{AR}$ experience more pronounced improvements in left ventricular function and remodeling than patients without anti- $\mathrm{AT}_{1}-\mathrm{AR}$ when given perindopril in combination with standard treatment over a shorter period of time. It should be noted that long-term clinical efficacy in patients with $(+)$ anti- $\mathrm{AT}_{1}-\mathrm{AR}$ was equivalent to that observed in patients without anti- $\mathrm{AT}_{1^{-}}$ 
$\mathrm{AR}$. It is here speculated that anti- $\mathrm{AT}_{1}-\mathrm{AR}$ might be a useful biomarker of over-activation of the renin-angiotensinaldosterone system. This is the first study to link anti$\mathrm{AT}_{1}-\mathrm{AR}$ to treatment strategies for congestive heart failure.

\section{Competing interests}

The authors declare that they have no competing interests.

\section{Authors' contributions}

LZ contributed to study design and drafting part of manuscript. All authors contributed to the acquisition of data and analysis of the results. QD, WJL, $\mathrm{WH}$, and LZ were involved in drafting the manuscript. All authors revised the manuscript critically and approved the final version.

\section{Acknowledgements}

We would like to extend our heartfelt thanks to the patients and their families whose participation made this project possible. We would also like to thank Professor Hui-rong Liu for her assistance in technical matters. This project was supported by the Natural Science Foundation of China (81250011, 81370340).

\section{Author details}

${ }^{1}$ Heart Failure Center, Departments of Cardiology, Capital Medical University, Chao-Yang Hospital, Beijing, China. ${ }^{2}$ Children's Hospital of Philadelphia Research Institute, University of Pennsylvania School of Medicine, Philadelphia, PA, USA.

Received: 16 December 2012 Accepted: 25 October 2013 Published: 31 October 2013

\section{References}

1. Wallukat G, Homuth V, Jupner A, Ruckert E, Haller H, Luft FC: Patients with preeclampsia develop agonistic autoantibodies against the angiotensin AT(1) receptor. Hypertension 1997, 30(4):123-123.

2. Wallukat G, Homuth V, Fischer T, Lindschau C, Horstkamp B, Jupner A Baur E, Nissen E, Vetter K, Neichel D, et al: Patients with preeclampsia develop agonistic autoantibodies against the angiotensin AT1 receptor. J Clin Invest 1999, 103(7):945-952.

3. Jin Z, Wang J, Zhang WH, Zhang GH, Jiao XY, Zhi JM: Changes in cardiac structure and function in rats immunized by angiotensin type 1 receptor peptides. Acta Biochim Biophys Sin (Shanghai) 2011, 43(12):970-976.

4. Wallukat G, Neichel D, Nissen E, Homuth V, Luft FC: Agonistic autoantibodies directed against the angiotensin II AT1 receptor in patients with preeclampsia. Can J Physiol Pharmacol 2003, 81(2):79-83.

5. Vantrimpont P, Rouleau JL, Wun CC, Ciampi A, Klein M, Sussex B, Arnold JM, Moye L, Pfeffer M: Additive beneficial effects of beta-blockers to angiotensin-converting enzyme inhibitors in the Survival and Ventricular Enlargement (SAVE) Study. SAVE Investigators. J Am Coll Cardiol 1997, 29(2):229-236

6. Atlas SA: The renin-angiotensin aldosterone system: pathophysiological role and pharmacologic inhibition. J Manag Care Pharm 2007, 13(8 Suppl B):9-20.

7. Enright PL: The six-minute walk test. Respir Care 2003, 48(8):783-785.

8. Fu ML, Herlitz H, Schulze W, Wallukat G, Micke P, Eftekhari P, Sjogren KG, Hjalmarson A, Muller-Esterl W, Hoebeke J: Autoantibodies against the angiotensin receptor (AT1) in patients with hypertension. $J$ Hypertens 2000, 18(7):945-953.

9. Ma G, Li Y, Zhang J, Liu H, Hou D, Zhu L, Zhang Z, Zhang L: Association between the presence of autoantibodies against adrenoreceptors and severe pre-eclampsia: a pilot study. PLoS One 2013, 8(3):e57983.

10. Zou C, Zhang Z, Zhao W, Li G, Ma G, Yang X, Zhang J, Zhang L: Predictive value of pre-procedural autoantibodies against M2-muscarinic acetylcholine receptor for recurrence of atrial fibrillation one year after radiofrequency catheter ablation. J Trans/ Med 2013, 11:7.

11. Liao YH, Wei YM, Wang M, Wang ZH, Yuan HT, Cheng LX: Autoantibodies against AT1-receptor and alpha1-adrenergic receptor in patients with hypertension. Hypertension research 2002, 25(4):641-646.
12. Voors AA, van Veldhuisen DJ: Pharmacological treatment of chronic heart failure according to the 2005 guidelines of the European Society of Cardiology. Minerva cardioangiologica 2005, 53(4):233-239.

13. Hunt SA, Abraham WT, Chin MH, Feldman AM, Francis GS, Ganiats TG, Jessup M, Konstam MA, Mancini DM, Michl K, et al: ACC/AHA 2005 Guideline Update for the Diagnosis and Management of Chronic Heart Failure in the Adult: a report of the American College of Cardiology/ American Heart Association Task Force on Practice Guidelines (Writing Committee to Update the 2001 Guidelines for the Evaluation and Management of Heart Failure): developed in collaboration with the American College of Chest Physicians and the International Society for Heart and Lung Transplantation: endorsed by the Heart Rhythm Society. Circulation 2005, 112(12):e154-e235.

14. Chabot A, Jiang BH, Shi Y, Tardif JC, Dupuis J: Role of aldosterone on lung structural remodeling and right ventricular function in congestive heart failure. BMC Cardiovasc Disord 2011, 11:72.

15. Zhang L, Cui L, Miao GB, Zhao WS, Wang SY, Liu XL: [Study of autoantibodies against the G-protein-coupled beta 2- and alpha 1adrenergic and AT1 receptors in patients with primary hypertension] Zhongguo Yi Xue Ke Xue Yuan Xue Bao 2002, 24(4):367-369.

16. Tepper D: A randomized trial of the angiotensin-receptor blocker valsartan in chronic heart failure. Congest Heart Fail 2002, 8(1):57-58.

17. Fazeli G, Stopper H, Schinzel R, Ni CW, Jo H, Schupp N: Angiotensin II induces DNA damage via AT1 receptor and NADPH oxidase isoform Nox4. Mutagenesis 2012, 27(6):673-681.

18. Yoshida H, Takahashi M, Tanonaka K, Maki T, Nasa Y, Takeo S: Effects of ACE inhibition and angiotensin II type 1 receptor blockade on cardiac function and $\mathrm{G}$ proteins in rats with chronic heart failure. $\mathrm{Br} J$ Pharmacol 2001, 134(1):150-160.

19. LaMarca B, Wallace K, Granger J: Role of angiotensin II type I receptor agonistic autoantibodies (AT1-AA) in preeclampsia. Curr Opin Pharmacol 2011, 11(2):175-179.

20. Dragun D, Muller DN, Brasen JH, Fritsche L, Nieminen-Kelha M, Dechend R, Kintscher U, Rudolph B, Hoebeke J, Eckert D, et al: Angiotensin II type 1 -receptor activating antibodies in renal-allograft rejection. $N$ Engl J Med 2005, 352(6):558-569.

21. Dechend R, Viedt C, Muller DN, Ugele B, Brandes RP, Wallukat G, Park JK, Janke J, Barta $P$, Theuer J, et al: AT1 receptor agonistic antibodies from preeclamptic patients stimulate NADPH oxidase. Circulation 2003, 107(12):1632-1639.

22. Chai W, Zhang W, Jin Z, Feng Y, Kuang Y, Zhi J: Angiotensin II type I receptor agonistic autoantibody-induced apoptosis in neonatal rat cardiomyocytes is dependent on the generation of tumor necrosis factor-alpha. Acta Biochim Biophys Sin (Shanghai) 2012, 44(12):984-990.

23. Zhang S, Zhang X, Yang L, Yan Z, Yan L, Tian J, Li X, Song L, Wang L, Yang $X$, et al: Increased susceptibility to metabolic syndrome in adult offspring of angiotensin type 1 receptor autoantibody-positive rats. Antioxid Redox Signal 2012, 17(5):733-743.

24. Cappuzzello C, Di Vito L, Melchionna R, Melillo G, Silvestri L, Cesareo E, Crea F, Liuzzo G, Facchiano A, Capogrossi MC, et al: Increase of plasma IL-9 and decrease of plasma IL-5, IL-7, and IFN-gamma in patients with chronic heart failure. J Transl Med 2011, 9:28.

25. Lanfear DE, Hasan R, Gupta RC, Williams C, Czerska B, Tita C, Bazari R, Sabbah HN: Short term effects of milrinone on biomarkers of necrosis, apoptosis, and inflammation in patients with severe heart failure. J Trans/ Med 2009, 7:67.

26. McMurray J, Davie AP: Angiotensin-(1-7) attenuates the development of heart failure after myocardial infarction in rats. Circulation 2002, 106(20):e147. author reply e147.

27. Chappell MC: Emerging evidence for a functional angiotensin-converting enzyme 2-angiotensin-(1-7)-MAS receptor axis: more than regulation of blood pressure? Hypertension 2007, 50(4):596-599.

28. Thai H, Wollmuth J, Goldman S, Gaballa M: Angiotensin subtype 1 rReceptor (AT1) blockade improves vasorelaxation in heart failure by up-regulation of endothelial nitric-oxide synthase via activation of the AT2 receptor. J Pharmacol Exp Ther 2003, 307(3):1171-1178.

29. Gao L, Zucker $H$ : AT2 receptor signaling and sympathetic regulation. Curr Opin Pharmacol 2011, 11(2):124-130.

30. Rossitto G, Regolisti G, Rossi E, Negro A, Nicoli D, Casali B, Toniato A, Caroccia B, Seccia TM, Walther T, et al: Elevation of angiotensin-II type-1- 
receptor autoantibodies titer in primary aldosteronism as a result of aldosterone-producing adenoma. Hypertension 2013, 61(2):526-533.

31. Stork S, Boivin V, Horf R, Hein L, Lohse MJ, Angermann CE, Jahns R: Stimulating autoantibodies directed against the cardiac beta1adrenergic receptor predict increased mortality in idiopathic cardiomyopathy. Am Heart J 2006, 152(4):697-704.

32. Zannad F, Agrinier N, Alla F: Heart failure burden and therapy. Europace 2009, 11(Suppl 5):v1-v9.

doi:10.1186/1471-2261-13-94

Cite this article as: Du et al:: Perindopril treatment promote left ventricle remodeling in patients with heart failure screened positive for autoantibodies against angiotensin II type 1 receptor. BMC

Cardiovascular Disorders 2013 13:94.

\section{Submit your next manuscript to BioMed Central and take full advantage of:}

- Convenient online submission

- Thorough peer review

- No space constraints or color figure charges

- Immediate publication on acceptance

- Inclusion in PubMed, CAS, Scopus and Google Scholar

- Research which is freely available for redistribution 